\title{
DAS STÜCK 》WANABNI« DER PALÄSTINENSERIN KAMILYA JUBRAN UND DES SCHWEIZERS WERNER HASLER IM MULTILOKALEN HÖRTEST. EINE MULTIPERSPEKTIVISCHE ANALYSE
}

\author{
Thomas Burkhalter, Christoph Jacke \\ und Sandra Passaro
}

\section{Einleitung}

Kamilya Jubran und Werner Hasler treffen sich zum ersten Mal 2002 in Bern. Werner Hasler, ein Schweizer Trompeter und Electronica-Künstler, ist fasziniert von der Tiefe und Vielfalt im Gesang von Kamilya Jubran sowie von ihrem künstlerischen Ehrgeiz und ihrer fordernden Art. Kamilya Jubran, die Sängerin und Oud-Spielerin der bekannten palästinensischen Gruppe Sabreen, ist auf der Suche nach einem neuen musikalischen Ausdruck in ihrer Musik. Sie will ihre Stimme und die Gedichte von arabischen Dichtern in neue musikalische Kontexte stellen. In Werner Hasler sieht sie ihren idealen musikalischen Partner (vgl. Interviews mit Jubran und Hasler 2002). Die beiden Musiker finden primär aus künstlerischen Interessen zueinander. Die kulturellen Märkte (mit ihren Agenturen, Labels, Förderern und Rezipienten) hingegen fokussieren immer wieder auf die zwei »Kulturen«, denen die beiden Musiker entstammen. Das wird dieser Artikel auch zeigen.

Jubran und Hasler treten bald in der Schweiz und in Ägypten auf. In Ägypten löst ihre Musik besonders zwiespältige Reaktionen aus: Ein Teil des ägyptischen Publikums stört sich an der Electronica und der neuen Art, in der Kamilya Jubran die Texte und Musik ägyptischer Musiklegenden wie Sayyd Darwish und Umm Kulthum umsetzt; der andere Teil - vor allem junge Musiker der experimentellen Szene - zeigt sich begeistert: Diese Musik sei modern und gleichzeitig sehr arabisch (vgl. Burkhalter 2003, 2006) Doch was genau heißt das? Ist sarabisch < überhaupt definierbar? Stehen Jubran und Hasler in einem >interkulturellen • Austauschprojekt zwischen 
Palästina und der Schweiz, zwischen der arabischen Welt (oder: dem Nahen Osten) und Europa, oder gar zwischen Orient und Okzident? Lässt sich ihre Musik folglich über das Konzept der »Alternativen Modernität« (Gaonkar 2001) deuten? Letzteres würde suggerieren, Jubran und Hasler schafften eine neue arabische Musik, d.h. sie wäre in ihrer Identität demnach >arabisch und Werner Hasler entweder ein geschickter, zurückhaltender Begleiter, oder aber ein Kenner und Könner der arabischen Musik. Oder aber täuschen wir uns? Musizieren Kamilya Jubran und Werner Hasler letztlich in einem ganz anderen Modus: einem trans-, hyper- oder gar popkulturellen Modus? Oder können wir dem Fokus auf »Kultur« entkommen?

Im Zentrum unseres Beitrags steht das Stück »Wanabni« von der gleichnamigen, 2010 erschienenen zweiten CD des Duos. Die Wahl dieses, laut dem Duo »wichtigen« und »repräsentativen« Stücks (Jubran 2010; Hasler 2010) erfolgte in Zusammenarbeit mit den beiden Musikern. Anhand des Stückes wollen wir ein multi-disziplinäres methodisches Forschungslayout zur Diskussion stellen, mit dem inter-, trans-, hyper- und popkulturelle Musik heute analysiert werden kann. »Wanabni« wird in unserer Studie anhand eines multiperspektivischen Forschungsdesigns untersucht. In diesem rekurrieren wir auf diverse anschließbare kulturtheoretische, musikethnologische und popmusikwissenschaftliche Theorien und Modelle. Davon ausgehend wollen wir in diesem Artikel Fragen an unser konkretes Fallbeispiel stellen und diese explorativ anhand von empirischen Methoden aus den Medienkultur-, Musik- und Sozialwissenschaften überprüfen. Wir konzentrieren uns dabei aus den notwendigen abstrakten theoretischen Rahmungen heraus auf eine Musikanalyse (sowohl von uns als auch von den beiden Musikern) und einen an die Studie angepassten multilokalen Hörtest (vgl. Tagg/Clarida 2003; Steinholt 2005; Burkhalter 2009), der Hörende aus verschiedenen Kulturen einbezog. Um den Erhebungs- und Forschungsprozess dialogisch zu gestalten und Korrekturen zuzulassen, haben wir uns für das dialogische Editieren im Sinne des Sound-Anthropologen Steven Feld (1982) entschieden.

\section{Theoretisch-method(olog)ische Rahmung der Studie}

»Diversität kann nicht mit einem normativen oder universellen, sondern nur mit einem sich selbst auch in Frage stellenden, also relativen Kulturbegriff abgebildet werden« (Binas-Preisendörfer 2010: 247). Zu diesem Fazit gelangt die deutsche Popmusikethnologin und Musikwissenschaftlerin Susanne 
Binas-Preisendörfer im Ausblick ihrer Studie zu Popmusik auf globalen Märkten und in lokalen Kontexten. Wollen wir uns einem derart Nationen und Kontinente übergreifenden, durch mediale Technologien transferierten und Stile vermischenden Phänomen wie der Musik von Kamilya Jubran und Werner Hasler nähern, so benötigen wir grundlegend einen an Prozess und Dynamik orientierten, Medien sensiblen, weiten, möglichst wenig normativen, reflexiven Kulturbegriff. Der Medienkulturwissenschaftler Siegfried J. Schmidt entwickelt diesen in Form seines Konzepts der »Kultur als Programm« seit den 1990er Jahren (vgl. Schmidt 1994 u. 2004: 70-107, 122125). Dabei wird Kultur als Interpretationsfolie für Wirklichkeitsmodelle aufgefasst, die über in jeweiligen Programmen zentrale Kategorien (z.B. Geschlecht, Raum, Zeit, Familie) mit affektiven Gewichtungen und moralischen Bewertungen versehen wird. Kultur erscheint also als dehnbare Universalie, die gewissermaßen hinter unseren Rücken unsichtbar abläuft: Es gibt keine Kultur, aber wir brauchen sie. Ohne eine Kultur, die sich ständig über Akteure vollzieht, würden keine Einordnungen, Interpretationen und Bewertungen von Wirklichkeit stattfinden. Im Sinne Schmidts, der sich in seinem soziokulturellen Konstruktivismus bemüht, Aspekte der Systemtheorie Niklas Luhmanns und der Handlungstheorie Jürgen Habermas' zu koppeln, laufen Verstehen und Handeln durch Akteure stets kognitiv autonom und sozial orientiert ab, entscheiden wir also subjekt- und kulturabhängig gleichermaßen. Dieses komplexe Modell, welches hier nur in aller Kürze skizziert werden kann, wurde für den Bereich von Populärkulturen und vor allem Subkulturen von Christoph Jacke (2004) weiter entwickelt und lässt sich an zahlreiche ähnlich verortete Konzepte von Popmusik-Kultur anschließen (vgl. etwa Thompson/Balkwill 2010), was bis dato allerdings für konkrete popkulturelle und popmusikalische Phänomene kaum praktiziert wurde. Eine derart abstrakte Rahmung erscheint angebracht, um in unserer Fallstudie dennoch Grundlagen und Anschlüsse zu schaffen.

In Schmidts und Jackes Begrifflichkeiten wäre Musik ein kulturelles Teilprogramm $^{1}$ mit Unterprogramm-Ebenen wie Jazz, Laptop-Electronica oder Tarab-Musik (vgl. Racy 2003) mit in sich kontradiktorischen Ebenen (Differenzen), „wobei Programme verstanden werden als spezifische, erlernte Regeln des Verhaltens« (Schmidt 1994: 225). Innerhalb dieser gibt es dann

1 Laut Schmidt bilden Kulturprogramme zusätzlich zu Teilprogrammen auch Subprogramme bzw. Subroutinen aus, die die Anwendungsresultate »einer Zweitoder Dritt-Codierung unterziehen im Hinblick auf beobachtungsleitende Differenzen« (Schmidt 2004: 84), in der Modifikation von Jacke (2004) wären dies Subkulturprogramme, die insbesondere und bis heute im Popmusikalischen auftreten. Auch hier zeigt sich die Tauglichkeit dieser Überlegungen für Kulturalitätsstudien. 
wiederum wichtige Kategorien wie etwa das musikalische Prinzip Maqam in der arabischen Tarab-Musik. Vom kulturellen Hauptprogramm einer Gesellschaft lassen sich so feinste Verästelungen bis ins Spezialistentum der musikalischen Nischenkulturen beobachten, die sich (bedingt) gegenseitig beeinflussen. Gleichzeitig wird Kultur als Universalie verstanden und somit entscheidend Vergleichbarkeit hergestellt. Besonders deutlich wird in diesem Modell, dass etwa Jazz, Electronica/Elektronische Laptop-Musik ('westlich< geprägt) und Tarab/Maqam (>östlich` geprägt) kultürlich unterschiedlich relevante (zentral/peripher) Kategorien des jeweiligen musikalischen Teilprogramms bedeuten und wiederum unterschiedlich gewichtet, bewertet und mit divergierenden Emotionen konnotiert werden.

Uns geht es hier nicht so sehr um eine präzise Einordnung des von uns untersuchten Phänomens in kulturelle Haupt- oder Subprogramme, also um die Einnahme einer intrakulturellen Perspektive, sondern darum, ein (neues) popmusikalisches Phänomen, das Zusammenspiel der palästinensischen Sängerin und Oud-Spielerin Kamilya Jubran mit dem Schweizer Jazz- und Elektronik-Laptop-Musiker und Trompeter Werner Hasler und ihre (auch gegenseitige) kulturelle (Selbst-)Rezeption überhaupt erst einmal vor einem popmusik- und kulturtheoretischen Hintergrund genauer zu analysieren und an Grundlagen anschließbar zu machen.

\subsection{Modi von Kulturalität}

Die Modi von Kulturalität werden mittlerweile in Philosophie, Medienkultur-, Musik-, Sozial- und Kulturwissenschaften intensiv beobachtet und beschrieben. Sie lassen sich in den Bereichen der Popmusik besonders gut erkennen. Es können folgende Modi aus diesen Forschungen herauskristallisiert werden, die für unsere Studie und daran anschließende Überlegungen praktikabel erscheinen, weil sie eben einerseits an die eigenen kulturtheoretischen Modelle koppelbar und andererseits aus konkreten Phänomenen heraus abgeleitet sind. Bei den verschiedenen Analysen wurden diese Modi soweit in der Kürze der Studie möglich - überprüft.

\subsubsection{Multikulturalität}

In diesem Modus laufen unterschiedliche kulturelle Haupt- und Subprogramme quasi nebeneinander her, stören oder irritieren sich nicht, sondern werden bestenfalls gegenseitig beobachtet und toleriert. In letzter Zeit wird dieser, speziell in den 1980er und 1990er Jahren bei Politikern beliebte Kulturalitäts-Modus wegen seiner nur vermeintlichen Flexibilität kritisiert, 
hinter der sich oftmals Konservativität und Abschottung bei gleichzeitiger temporär oder räumlich begrenzter Duldung (das multikulturelle Stadtteilfest) verbirgt, aber keine Integration oder Inklusion erfolgt (vgl. kritisch dazu etwa Terkessidis 2008, 2010²; Žižek 2003).

\subsubsection{Interkulturalität}

Im Modus der Interkulturalität berühren sich demgegenüber die unterschiedlichen kulturellen Programme bzw. kommunizieren ihre >Programmanwender < miteinander. Kontakt wird hergestellt und betont, Differenzen überhaupt erst einmal wahrgenommen und reflektiert. Dieser Modus bedeutet also einen kommunikativen Schritt in Richtung Verständigung, erreicht aber nur Aufmerksamkeit, keine Änderung in den jeweils eigenen Programmen und also Lebensformen (vgl. etwa Rosengren 2002; Terkessidis 2010). Der Philosoph Wolfgang Welsch betont die durchaus produktiven, kommunikativen Aspekte dieses Modus:

»Das Interkulturalitätskonzept ist zweistufig. Auf der Primärebene geht es von wohl abgegrenzten und beträchtlich verschiedenen Kulturen aus; auf der Sekundärebene fragt es dann, wie diese Kulturen sich miteinander vertragen, wie sie einander ergänzen, wie sie miteinander kommunizieren, einander verstehen oder anerkennen können« (Welsch 1994: 149).

Multi- und Interkulturalität sind also >kulturprogrammlich erste Schritte aufeinander bzw. auf das jeweils Fremde, Andere zu, aber keinesfalls dauerhaft ineinander greifend und sich gegenseitig modifizierend. Das Konzept der >Alternativen Moderne < kann diesem Modus zugefügt werden. Gemeint ist eine >Moderne<, die nicht die Musik aus dem Westen kopiert, sondern aus der eigenen Kultur schöpft, sie neu formuliert, oder gar neu erfindet. »Alternative Modernität« (Gaonkar 2001) impliziert eine Standard-Modernität im Westen und Alternativen dazu im Nicht-Westen. ${ }^{3}$

2 Mark Terkessidis (2010: 111-168) verwendet in Ablehnung des eher konservativen Multi- und Interkulturalitätsbegriffs die Bezeichnung »Interkultur « als notwendige Bedingung für Musterveränderung und Barrierefreiheit.

3 Diese Anordnung ist allerdings aus verschiedenen Gründen fragwürdig: Erstens weisen Wissenschaftler heute immer deutlicher darauf hin, dass Modernität immer im Zusammenspiel des sGlobalen Nordens - mit dem 'Globalen Süden entstanden ist (vgl. Randeria/Eckert 2009). Zweitens sollte -Kultur < nicht essentialistisch definiert sein. Die globalisierte Welt kennt schon lange keine scharfen Trennungen mehr zwischen seigen< und 'fremd<, söstlich < und >westlich<. 


\subsubsection{Transkulturalität}

Wolfgang Welsch definiert diesen Modus wie folgt:

"An die Stelle der Kulturen alten Zuschnitts - die man sich immer als eine Art National- oder Regionalkulturen vorgestellt hat - sind heute diverse Lebensformen getreten. Diese Lebensformen (nach meiner Auffassung: die Kulturen von heute, die Kulturen nach dem Ende der traditionellen Kulturen) machen nicht an den Grenzen der alten Kulturen halt, sondern gehen quer durch diese hindurch. Deshalb sind sie mit den herkömmlichen Kulturkategorien nicht mehr zu fassen. >Transkulturalität < will beides anzeigen: dass wir uns jenseits der klassischen Kulturverfassung befinden; und dass die neuen Kultur- bzw. Lebensformen durch diese alten Formationen wie selbstverständlich hindurchgehen « (Welsch 1994: 147f., Hervorhebungen im Original).

Mit Welsch ist neben Gestaltung im Sinne von Design und Wissenschaft vor allem Musik eine transkulturelle Lebensform. Nach Schmidt und Jacke wäre Musik ein transkulturelles Programm, welches insbesondere in seiner Ausprägung als Popmusik quer zu an Nationen gebundenen Hauptprogrammen oder gesellschaftlichen Systemen verläuft. Musik liefert somit ihre eigenen Orientierungen, Einordnungen, Interpretationen und Bewertungen, bildet selbst aber (noch) kein umfassendes, vertikales Hauptprogramm einer Kultur, sondern verläuft eher horizontal. Musik bewirkt in diesem Sinne aber durchaus in den vertikalen Programmen Veränderungen, nur eben (noch) nicht umfassend, weswegen Pop ja auch seinen oftmals spielerischen oder Übungs-Charakter behält und selten etwa gesellschaftsgefährdend und tatsächlich revolutionär wirkt. ${ }^{4}$ Hier bilden sich mit Welsch also neue, integrative Lebensformen um Popmusik herum aus, die nicht mehr zwingend an Nationen orientiert sind, was aber gleichzeitig keine Willkür oder komplette Abschottung (mehr) von traditionellen Kulturen bedeutet (vgl. Binas-Preisendörfer 2010: 75-109).

\subsubsection{Hyperkulturalität}

Neuerdings werden (medien-)kulturtheoretische Konzepte entwickelt, die nunmehr die klar erkennbaren Wurzeln und Quellen von kulturellen Hauptprogrammen nicht mehr identifizierbar erscheinen lassen und die vollkommen neu orientierten, auf den horizontalen Ebenen vernetzten (Teil-)Pro-

4 Vgl. etwa Hepp 2006, Jacke 2004, Schmidt 2004: 100-104, Terkessidis 2008, 2010, Welsch 2010 und für Popmusik Meyer 2007 sowie bereits Wallis/Malm 1987. 
gramme ins Auge fassen. Dabei meint Hyperkulturalität nicht Anarchie oder Unverbindlichkeit, sondern betont kulturelle und auch popmusikalische Entwicklungen, die sich aus anderen Quellen speisen, nicht aber zwingend deren Ursprünge kulturprogrammlich erlebt haben oder nachvollziehen können bzw. wollen (vgl. etwa Han 2005; Terkessidis 2010). Hier wird gegenüber der Transkulturalität gar nicht mal mehr das jeweils Andere, Neue bewusst durchdrungen und Gemeinsames im Fremden gesucht. Man bedient sich vielmehr aus zumeist freien Angeboten, um bestimmte Effekte zu erzielen und pragmatisch zu inkludieren.

\subsubsection{Superkulturalität}

Eine sehr auf Popmusik bezogene Variante der Hyperkulturalität hat der USamerikanische Kommunikations- und Popmusikforscher James Lull (1992, 2000: 264-276, 2002) durchaus kritisch als Superkultur bezeichnet, in der bestimmte weltweit über Medien erreichbare und erhältliche Angebote verteilt werden und so - zunächst unabhängig von lokalen Spezifiken - global distribuiert werden. Große Werbemarken aber auch weltweite PopmusikStars werden als Beispiele genannt: »Superculture: A personalized matrix of symbolic forms, unmediated everyday scenes, and all other available cultural representations and activities« (Lull 2000: 291). Man könnte den Modus der »Superkulturalität« als eine kommerzialisierte, auf Massenkonsum ausgerichtete Mischung aus den vorhergehenden Modi bezeichnen, die alle kulturellen Produkte, Stile und Habitus >vor Ort < kommerziell nutzend vereint und inkludiert. Dieser Modus wird hier eher zu vernachlässigen sein, da wir uns einem sehr spezifischen und Nischenphänomen jenseits des erfolgreichen Mainstreams bzw. >Blockbustertums< nähern wollen.

Wenn auch bisher so nicht bezeichnet, ließe sich vor allem für Forschungen zur Popkultur und ihrem Nukleus Popmusik für diesen übergelagerten Modus der Begriff der »Popkulturalität« nutzen, der eben verdeutlicht, dass hier zum einen keine umfassenden, traditionell gewachsenen Kulturprogramme mehr vorherrschen und dass zum anderen teilweise nicht mehr identifizierbare Ursprünge, weltweite, medial leicht erreichbare Einflüsse und lokale Verbindlichkeiten spielerisch und künstlerisch vermengt werden, oftmals aus sowohl kommerziellen als auch experimentellen Beweggründen heraus, was den Modus von dem der Hyperkulturalität unterscheidet. ${ }^{5}$ Die-

5 In der populären Musik gibt es diese Vermengungen von Anfang an, wie Peter Wicke (2001) bereits für die Zeit der Wiener Tanzsalons und des Walzers im 18./19. Jahrhundert konstatiert. Vgl. aktuell zu popmusikkulturellem Crossover exemplarisch an der Musikerin Cibelle aus journalistisch-essayistischer Perspek- 
ser Begriff ist dem der »Cross-Kulturalität « im Verständnis der Musikpsychologen Thompson/Balkwill (2010) ähnlich, die in ihrer Studie kulturelle 'Ladungen< und cross-kulturelle Effekte im Zusammenhang mit Musik und Emotion untersucht haben; ${ }^{6}$ dieses allerdings eher quantitativ-repräsentativ und mit der Forderung nach mehr explorativ-qualitativer Forschung für cross-kulturelle Unterschiede und Gemeinsamkeiten in der Rezeption und Nutzung von Musik, die wir hier einzulösen versuchen. ${ }^{7}$

\subsection{Forschungsfragen}

Diese Modi von Kulturalität, die sich in dem der Popkulturalität vereint finden, gilt es, in unserer Studie fallbeispielhaft zu überprüfen bzw. zu analysieren. Es ergeben sich daher für die Analyse folgende Fragestellungen:

1. Wieweit sind im Stück »Wanabni« Prinzipien und Bedeutungen aus Tarab/Maqam, Jazz und der elektronischen Musik noch vorhanden? Wieweit entfernen sich die Jubran und Hasler von diesen »Traditionsströmen « im Sinne von Assmann (1992)? ${ }^{8}$

2. Was für einer Art kulturellem Programm (oder was für Programmen) entspringt »Wanabni «? Oder entstehen in der Rezeption multiple Bedeutungen und Missdeutungen? - Möchten Kamilya Jubran und Werner Hasler zum Beispiel eine persönliche, popkulturelle Musik schaffen, werden aber immer als interkulturelles Projekt zwischen >Orient< und >Okzident wahrgenommen?

tive Diederichsen 2010, vgl. zur Deterritorialisierung von Popmusik durch Laptop-Künstler Cascone 2003 und zur Popkultur als prototypischem Feld von Glokalisierung im Sinne gleichzeitiger globaler kultureller Verschmelzung und lokaler Differenz-Setzung Klein/Friedrich 2003.

6 Freilich sind auch derartige Festlegungen wiederum kulturell geladen und dementsprechend vorgeprägt. Hier zeigt sich die Notwendigkeit eines reflexiven Kulturbegriffs ganz deutlich: »How might we conduct a cross-cultural study of emotion and music without the conclusions being corrupted by cultural biases? « (Thompson/Balkwill 2010: 755).

7 Anschlüsse zeigen sich vor allem an aktuelle Diskurse der Globalisierung von Kultur unter besonderer Berücksichtigung nicht-westlicher Perspektiven, wie sie etwa von Arjun Appadurai (2003, 2009) und Ghassan Hage (2003) oder in Bezug auf Hybridität von Kien Nghi Ha (2005) sowie speziell für die Popmusik und ganz ähnlich unserer Studie zuletzt von Richard A. Peterson/Andy Bennett (2004) zu Musikszenen, Burkhalter (2009) zur Popmusik in Beirut, Johannes Ismaiel-Wendt (2010) zu popmusikalischen postkolonialen Topographien und von Thomas Solomon (2009) zu türkischem HipHop angestoßen wurden.

8 Für den Bereich der Popmusikforschung arbeiten mit dem Assmann'schen Konzept des Traditionsstroms u.a. Elflein (2010) sowie Ahlers/Jacke (2010). 
Die musikalische Kooperation der beiden Musiker unseres Fallbeispiels dient dabei als konkrete >Micro Case Study < vor abstraktem Hintergrund, um die geschilderten Beeinflussungen und Verästelungen zu untersuchen, und zwar sowohl am musikalischen Material als auch auf allen Stufen des popmusikalischen Kommunikationsprozesses (vgl. Jacke 2009; Jacke/Passaro 2011).

\subsection{Methoden}

Mit diesem hoch abstrakten, flexiblen und interdiskursiv anschließbaren theoretischen Gerüst wollen wir der für unsere Belange grundlegenden Forderung der schon zitierten Popmusikforscherin Binas-Preisendörfer entgegen kommen: „Eine wissenschaftliche Auseinandersetzung mit den musikalischen Phänomenen einer modernen globalisierten und mediatisierten Welt verlangt sowohl reflexive theoretische Konzepte als auch sehr konkrete, kleinteilige Studien« (Binas-Preisendörfer 2010: 103).

Die Methodik für diesen Artikel setzt sich zusammen aus eigenen Interviews mit Kamilya Jubran und Werner Hasler, eigenen Musikanalysen, und der Auswertung einer Befragung mithilfe eines Fragebogens, der an den Kategorien des musikalischen Wissens von Philip Tagg (2009: 4) orientiert war (siehe Anhang).

Den Fragebogen schickten wir per E-Mail an 45 ausgewiesene Testhörerinnen und -hörer aus unterschiedlichen geographischen Kontexten und aus allen professionalisierten Bereichen des Kommunikationsprozesses Popmusik von Produktion (z. B. Musiker, Produzenten) über Distribution (z. B. PR/Werbung, Marketing) zu Rezeption (z.B. Hörer) und Weiterverarbeitung (z.B. Journalisten, Wissenschaftler). Von 34 Testhörern haben wir längere und kürzere Antworten erhalten. Wir wollten feststellen, inwiefern in den verschiedenen kulturellen Modi gehört, eingeordnet, interpretiert und auch bewertet wird, und zwar sowohl intrakulturell (eben als Musiker, Produzent, Promoter, Vertrieb, DJ, Journalist, Veranstalter oder auch Wissenschaftler) als auch geographisch übergreifend (»multi-sited« im Sinne von Marcus 1995) zwischen den Kontinenten im Sinne des Transnationalen (vgl. Appadurai 2003, 2009; Solomon 2009). ${ }^{9}$ Die ausgewählten Hör-Testenden gelten uns als »Key Informants and Gatekeepers« im Sinne von O'Reilly (2009: 132-137), die wir über unsere langjährigen Erfahrungen problemlos kontaktieren und einbeziehen konnten, offen, voraussetzungsvoll und gleichzeitig kritisch an

9 Vgl. rahmend zu den transnationalen Entwicklungen der Popmusikindustrien Gebesmaier 2009 und Rutten 1994, der eine hilfreiche Typologie zu den Verhältnissen zwischen lokalen Musikszenen und nationalen Tonträgermärkten erarbeitet hat sowie die explorative Fallstudie von Jacke/Passaro 2011. 
den Tests teilzunehmen ${ }^{10}$. Alle Testhörer erklärten sich bereit, mit ihren Namen im Artikel zu erscheinen. Für unseren methodischen Ansatz erscheint uns dies entscheidend: Der Leser muss nachvollziehen können, wer aus welcher Perspektive über die zu analysierende Musik schreibt. Wichtig ist uns dabei zudem die Einbringung unserer eigenen popmusikalisch reflektierten Positionen im Sinne eines vorhandenen, aus Erfahrung generierten Expertentums (Musiker, DJ, Veranstalter, Promoter, Journalist, Wissenschaftler) und einer Art von autoethnograpischer Einbindung (vgl. Muncey 2010). Einen gekürzten Fragebogen haben wir auch an Kamilya Jubran und Werner Hasler gesendet - und wir haben sie in einem zweiten Schritt mit den Resultaten des Hörtests konfrontiert und sie direkt interviewt. Die Musiker konnten also in zwei Phasen Stellung nehmen.

\section{Die Analysen}

Wir möchten nun diejenigen Analysen und Hörer-Antworten vorstellen, die uns zur Beantwortung der Forschungsfragen dieses Artikels (siehe 2.2) am relevantesten erscheinen. Eine breitere Auswertung ist in Planung. Auf eine Analyse des Songtextes und eine Transkription des Stückes als Notentext verzichten wir bewusst. Der gewählte Maqam-Modus, in dem »Wanabni « steht, ist zwar inspiriert durch den Inhalt des Songtextes. Für unsere Analyse ist die Frage, warum welcher Maqam ausgewählt worden ist, jedoch nicht entscheidend. Entscheidend ist, ob Kamilya Jubran und Werner Hasler überhaupt Maqam-Prinzipien folgen oder nicht - und wie sie das tun. Auch eine Abbildung des Tracks in Notenschrift bringt für unsere Analyse wenig. Erstens, weil die Musiker »Wanabni« improvisatorisch erarbeitet haben und etwa in der Live-Umsetzung ständig variieren. Zweitens, weil unsere Forschung auch für einen musikwissenschaftlich nicht gebildeten Leser verständlich bleiben soll. Unsere Forschung soll für andere Forschungsperspektiven anschließbar sein.

\subsection{Musikanalyse}

»Wanabni « ist an sich ein transnationales, ein durch Nationen und Kulturen gereichtes Medienprodukt: Die gleichnamige CD wurde im Influx-Studio in Bern von Dave Muther aufgenommen. Philippe Tessier du Cros mischte die Aufnahme in den Boxon-Studios in Paris. Gemastert wurde die CD im jRaph-

10 Ein Manuskript der ausführlichen Version dieses Artikels ging bereits zur Überprüfung an alle Testhörer. 
Studio von Raphael Jonin und Philippe Tessier. Kamilya Jubran und Werner Hasler waren mit dabei.

Das Stück »Wanabni« ist 8:49 Minuten lang, gegliedert in fünf Hauptteile von unterschiedlicher Länge: zwei Instrumental-Teile (hier: Intro und Outro) und drei Gesangsteile. Kurze instrumentale Zwischenteile verbinden diese Hauptteile. Die Länge der Gesangsteile richtet sich nach der Länge der in hocharabisch gesungenen Textstrophen. Rhythmisch beginnt das Stück frei und mündet schließlich in einem Zyklus aus acht Schlägen. Das Stück basiert auf sieben tragenden Elementen: Der Stimme von Kamilya Jubran; der Trompete und der arabischen Laute Oud, die beide mit der Software »Guitar Rig« manipuliert sind, einem Bordun-Ton und einem pulsgebenden Signalsound - beides Mischungen aus gesampelten »Ummm« und »Ahh« von Kamilya Jubran, bearbeitet in der Software »Reaktor «; einem Oberton-reichen Synthesizer-Sound, produziert in »Reaktor «; und einem Bass, der über ein Orgel-Soundmodul kreiert wurde.

Einige Schlüsselmomente aus »Wanabni « sollen in der Folge detaillierter beschrieben werden. Die Analyse fokussiert dabei hauptsächlich auf diejenigen Parameter, die später in den Hörtestantworten diskutiert werden. ${ }^{11}$

Auszug 1: Den Auftakt macht die Trompete (0:00-0:0512), die sehr bald über einen Bordun-Ton (ein temperiertes $a-1$. Tonebene) gespielt wird. Kamilya Jubran setzt mit ihrer Stimme relativ unvermittelt ein (1:22). Sie zieht enge melodische Linien um den Zentralton $h$ (2. Tonebene) (sie zieht immer wieder auf ein $b$, das allerdings um einen Viertelton erhöht klingt), halb rezitierend, halb singend, und tritt in einem relativ freien Dialog mit der Trompete.

Auszug 2: Ein Signal-Sound setzt ein (2:52), der unregelmäßig zwischen dem linken und rechten Audio-Kanal hin und her wandelt. Hinzu stößt ein Synthesizer-Sound (3:00), der relativ frei, länger anhaltende, obertonreiche Töne auf $a, g, f$ und $e$ intoniert. Jubran setzt wiederum rhythmisch frei ein (3:33), kreist jetzt aber um einen höheren Zentralton, ein erhöhtes $c-$ was sich als dritte Tonebene lesen lässt. Sie pendelt mit ihrer Stimme zwischen diesem erhöhten $c$ und einem $d$.

Auszug 3: Der Orgelbass setzt bei 4:40 mit einer sich laufend, aber minimal verändernden rhythmischen Struktur ein. Jubran singt ab 5:02 wieder um den Zentralton $h$ (zweite Tonebene), jedoch in schnelleren melodischen

11 Das ganze Stück und die unten beschriebenen Auszüge (1-4) können auf der Projektwebseite http://norient.com/academic/wanabni nachgehört werden. Auf dieser Projektwebseite befinden sich zudem der Songtext sowie Videos und Fotos der Musiker.

12 Die Zeiten beziehen sich auf den gesamten Track, nicht auf die Ausschnitte. 
Phrasen, die auch höhere Töne ansteuern als in allen Teilen zuvor - zum Beispiel das oktavierte $a$ (erste Tonebene).

Auszug 4: Relativ unvermittelt setzt danach die Oud ein (6:35) mit einem perkussiv gespielten Intro mit Trillern zwischen $e$ und $f$ (Tonebenen 5 und 6) und später auf $a$ (erste Tonebene), rhythmisch orientiert an der Struktur der Bassorgel. Die Synthesizer-Sounds werden über ein Chaos Pad (Kaoss Pad) manipuliert. Sie treten in einen engen, frei improvisierten Dialog mit der Oud - ähnlich dem Dialog zwischen Trompete und Gesang am Anfang von »Wanabni .

Diese erste, rein musikanalytische Perspektive auf »Wanabni« suggeriert, dass es sich hier nach den in Kapitel 2 erläuterten Parametern um ein interkulturelles Projekt handelt und der Ansatz der »Alternativen Modernität« nach Gaonkar (2001) greift: »Wanabni« nutzt Skalen und Prinzipien der Maqam-Musik (s.u.) - auf der einen Seite. Auf der anderen stehen Prinzipien und Instrumente, die wir aus dem weiten Umfeld von Jazz und Electronica kennen. Das Arrangement, das langsame Erarbeiten von kleinen Tonräumen, die improvisatorischen Momente und das improvisatorische Interplay zwischen Hasler und Jubran erinnert dabei aber stärker an die arabische Ensemble-Musik Tarab, wie sie detailliert von Jihad Racy (2003) beschrieben worden ist - als an Arrangements und Abläufe in Jazz und Electronica.

Das Maqam-Phänomen, ausführlich beschrieben von Habib Hassan Touma (1998), scheint den Kern dieser Musik auszumachen. Laut Touma (1998: 6474) beeinhaltet jeder Maqam:

1) eine Grundskala - oft mit Vierteltonhöhen.

2) Regeln, wie sich die Musik melodisch entwickelt (in klar getrennten Melodiezügen von tiefen in hohe Tonebenen und zurück).

3) Skalentöne, die neue Tonebenen eröffnen. (Die Musiker zirkulieren in engen Tonschritten um diese Tonebenen.)

4) Eine relativ freie rhythmisch-zeitliche Gliederung.

5) Einen ihm zugeordneten Gefühlszustand (Trauer, Stolz, Glück, etc. $)^{13}$

Gerade weil »Wanabni« vielen dieser Maqam-Prinzipien folgt, scheint die Kategorisierung »interkulturell« aber zu kurz zu greifen. Das Stück unterscheidet sich von vielen anderen Projekten, die zwischen Electronica/Jazz und nicht-westlicher Musik pendeln. Oft produzieren diese nämlich eine essentialistische, multi-kulturelle Hybridität (vgl. Ha 2005; Pfleiderer 1998):

13 Hier zeigt sich kulturelle Programmierung bzw. Lagerung besonders deutlich in der jeweiligen emotionalen Zuordnung. 
hier Europa als >moderner< elektronischer Grundbeat (und Basis), dort Asien als (pseudo-)traditionelles Ornament. Der Musikethnologe Jonathan Holt Shannon (2006: 67) liest dieses Basis-und-Ornament-Moment auch als Machtgefälle.

\subsection{Die Perspektiven von Werner Hasler und Kamilya Jubran}

Werner Hasler und Kamilya Jubran wussten nichts von unserer Einordnung des Stücks »Wanabni « in Richtung Maqam, als wir sie zum ersten Mal einzeln befragten (Hasler 4.8.2010, Jubran 11.8.2010). Bei beiden Gesprächen nutzten wir Ausschnitte aus dem Fragebogen als Leitfaden.

\subsubsection{Werner Hasler}

Werner Hasler widerspricht unserer Musikanalyse und unseren Einschätzungen (vgl. Kapitel 3.1) in verschiedenen Punkten. Für ihn steht »Wanabni« ebenso nah bei Jazz/Electronica wie bei Tarab/Maqam. "Wanabni« ist für ihn ein gleichberechtiger Dialog zwischen zwei Musikern. ${ }^{14}$ "Schlussendlich muss die Musik einfach klingen«, sagt Hasler und ist hier ganz Musikant und kein >verkopfter Theoretiker. Als zentrales Element in »Wanabni« nennt er den langen Spannungsbogen. Die Schwierigkeit ist, dass diese Spannung kontinuierlich und langsam aufgebaut wird, aber nicht abbricht. Zentral ist für Hasler in der Arbeit mit Kamilya Jubran aber auch die Suche nach anderen musikalischen Prinzipien und Formen - zum Beispiel nach neuen Skalen. Er ist deshalb ebenso interessiert an arabischen Skalen wie an den ZwölftonTechniken und Ganzton-Skalen von Arnold Schönberg und Olivier Messiaen.

\subsubsection{Kamilya Jubran}

Kamilya Jubran sieht für die Arbeit mit Werner Hasler eine zentrale Grundvoraussetzung: Die Musik ist ohne Druck von Außen und ohne Auftrag entstanden: »Es geht darum, live und in Echtzeit zwischen Gesang, Oud, Trompete und Elektronika zu interagieren«, betont sie. Bei der Frage nach den Melodien, Rhythmen und Formen nennt Jubran kulturelle Referenzen. Sie beschreibt ihren Gesang auf »Wanabni« als sehr minimalistisch. Das Singen

$14 \mathrm{Im}$ Grunde trifft auf unser popmusikalisches Beispiel genau das selbe zu, was der Journalist Jan Freitag (2011: 70) in seiner Rezension des aktuellen Albums der bekannten indisch-britischen Band Cornershop in der Wochenzeitung Die Zeit so treffend analysierte: »Hier geht es um Dialog; hier kommunizieren die Einflüsse, statt sich nur gegenseitig zu kontrastieren wie im Pop üblich.« 
über einen klaren emotionalen Modus über eine solch lange Zeit sieht sie als Anlehnung an die Vokalstile aus den Bergregionen von Groß-Syrien (Bilad al Sham) - eine Region um Jordanien, Libanon, Syrien, Palästina/Israel und Teile Iraks. Diese Region verbindet ähnliche kulturelle Praktiken. Eine davon ist der typische Ataba-Gesangsstil, der am besten bekannt ist durch die libanesischen Sänger Wadi Al Safi und Sabah. ${ }^{15}$

Die Musiker widersprechen in ihren Aussagen unserer ersten provisorischen Wertung von »Wanabni« als »interkulturelles Projekt« und als Ausdruck von »Alternativer Modernität«. Die Testhörer sollen uns weiterhelfen.

\subsection{Die Antworten der Experten aus dem Hörtest}

In diesem Abschnitt präsentieren wir eine Auswahl der Antworten aus dem Hörtest. Die Namen und Qualifikationen der Hörtest-Expertinnen und Experten sind in Anhang 2 vollständig aufgelistet. 95\% Prozent der Probanden werten »Wanabni« als spannend und interessant. 5\% Prozent stufen die Musik als eine kommerzielle Annäherung ein oder mögen sie gar nicht. Die Experten für arabische Musik sprechen von einem »attractive play between the spaces of >East and, West« (Martin Stokes), von einer »unusual and unfamiliar music, where you are curious to see how it develops « (Guilnard Moufarrej), und von einer »sensation that this music speaks to you and challenges you at the same time « (Rastegar). Martin Stokes, Musikethnologe und Experte für arabische Musik (vgl. Stokes 1992 und 2000), schreibt: »It engages, poses questions, does unusual and unexpected things. "Der Musiker, Journalist und Wissenschaftler Roger Behrens fragt, ob es »vielleicht der Versuch ist, im Gerüst von Elektronik traditionelle Musik und Jazz gleichermaßen zu retten?«

Jubrans Stimme wird von dem meisten gelobt und als authentisch, original und überzeugend empfunden. Der Musiker und Produzent Robert Koch erklärt: »| think its authentic. « Der Musikwissenschaftler und Kenner der arabischen Musik Neil van der Linden wertet Jubrans Gesang als eigenständig und nimmt einige Diskussionspunkte vorweg:

»Kamilya Jubran has a singing and composing style of her own, which she accentuates by choosing her own kind of accompaniment, on this album with electronic music. Of course there are side-references to certain musical traditions in the Arab world. Her voice has been justly compared to notably the Lebanese and Syrian Christian singing traditions, of which the Lebanese singer Fairuz is the most famous. But partly through the type of songs and

15 Vgl. dazu vgl. Burkhalter 2009, Stone 2008, Weinrich 2006, Asmar 1999. 
the accompaniment, she follows her own path and stays away from mainstream.«

Zu den Abschnitten musikalische Performance, Produktion und Interpretation äußern sich nur wenige Hörtest-Personen, ${ }^{16}$ wenn, dann aber durchwegs positiv. Der Popmusikforscher Michael Rappe schreibt: »Die MusikerInnen benutzen ihre Instrumente auf höchsten Niveau.« Robert Koch gefallen zudem der dynamische Umfang der Produktion und die Klangtexturen, die er als »warm« und »analog sounding « beschreibt.

\subsubsection{Musikalische Referenzen}

Die allermeisten Probanden nennen die Begriffe »arabisch« und »elektronisch«, um »Wanabni« zu umschreiben. Die Experten für arabische Musik nennen spezifischere Konzepte und Genres wie "palästinensische Musik « und »Bilad al Sham«. Die Experten für Pop und Elektronische Musik nennen oft den Begriff »Weltmusik«. Weitere Assoziationen sind hier nach Häufigkeit der Antworten sortiert: »Jazz« oder »NuJazz« oder »Acid Jazz«, »Ambient«, »orientalisch«, »Filmmusik«, »Trip Hop«, »Eastern«. Interessante Einzelnennungen sind: »exotisch «, »experimentell «, »Ethno und Genres wie »Folklore «, »Electronica«, »Space Rock«, »Krautrock«, »Dubstep«. Als Referenzen werden weiter einzelne Künstler angegeben: Pink Floyd, Bach, Brian Eno, Laurie Anderson, Deep Forest, Mille Plateaux, Matthew Herbert, Tied \& Tickled Trio, Nils Petter Molvaer, Uri Caine, Shabat Night Fever, Stockhausen, King Tubby, Lee Perry, Tricky (Track: »Overcome« vom Album Maxinquaye), Miles Davis, Markus Stockhausen, Henning Berg, und Mercan Dede. Florian Sievers, Redakteur des bekannten deutschen Magazins für elektronische Musik Groove, erwähnt Omar Faruk Tekbilek und das Projekt Sultan (auch bekannt als Peter Kuhlmann \& Burhan Öcal). Als Referenzmusiker aus der arabischen Welt, der Türkei und Aserbeidschan werden genannt: Dounia Yasin, Rima Banna mit Henrik Koitzsch, Abed Azrie, Anouar Brahem, Soap Kills, Ziyad Rahbani, Djivan Gasparjan und Badawi.

Zwei Hörer machen einen direkten Link zur Musik des Bilad al Sham dieselbe Musik, die auch von Kamilya Jubran als Referenz genannt wurde. Martin Stokes hört das Ataba-Genre als eine zentrale Einflussgröße. Ataba wird vom Musikethnologen Jihad Racy ausführlich diskutiert. Das Genre ist geprägt durch einen oft als emotional beschriebenen Gesang über klar definierte Reimstrukturen (vgl. Racy 1996: 411). Mustafa Said, Oud-Spieler und

16 Als Grund wird angegeben, dass eine genaue Analyse der Produktion sehr zeitaufwändig sei. 
Sänger aus Ägypten, situiert »Wanabni« zwischen der Folklore des Bilad al Sham (Ataba), religiösem Gesang, elektronischer Musik und Free Jazz.

Die genannten Referenzen sind extrem breit. Jeder Hörer scheint »Wanabni« aus seiner ganz eigenen Perspektive zu hören und dabei ständig Anknüpfungen zu suchen an die eigene Hörerbiographie. Eindeutig auszumachen sind aber die groben Hauptreferenzen Electronica, Jazz, Weltmusik und Ataba. Die Referenz Tarab/Maqam taucht bei den Testhörern erst bei der Frage nach einer genaueren Analyse von Melodien, Rhythmen und Formen auf.

\subsubsection{Melodien, Rhythmen, Formen}

Den Fragen nach Melodien, Rhythmen und Formen haben sich viele Expertinnen und Experten sehr genau angenommen - im Gegensatz etwa zu den Fragen zur Produktionsästhetik. Der Popmusik-Wissenschaftler Benjamin Schäfer spricht für viele, wenn er schreibt, dass »Wanabni« nicht wie ein 'westlicher Song strukturiert ist, sondern sich frei fließend durch Schichtungsverfahren entwickelt. Einige Kenner der arabischen Musik suchen nach dem spezifischen Maqam, in dem »Wanabni « steht. Martin Stokes hört »Wanabni « als eine Art Improvisation in den Modi Maqam Hijaz und Maqam Saba $^{17}$ (siehe dazu 4.1), erkennt dann aber selber, dass diese Kategorisierungen beim genauen Hören nicht ganz aufgehen - er bewertet das ausdrücklich als interessant. Der Musiker und Literaturwissenschaftler Kamran Rastegar und der ägyptische Oud-Spieler Mustafa Said teilen seine Einschätzung.

\subsubsection{Klassifizierung arabisch - europäisch-amerikanisch}

Die Antworten auf die Frage, ob die Hörerinnen und Hörer »Wanabni« als arabisch, europäisch oder amerikanisch hören, ergeben ein extrem breites Spektrum: von typisch arabisch zu typisch euro-amerikanisch, zu einer $\mathrm{Mi}$ schung aus arabisch und euro-amerikanisch, zu weder arabisch noch euroamerikanisch. Der Performer und Produzent Paul Bonomo und der Schauspieler und Musikrezipient Michael Wentzlaff finden, dass das >Arabische und das >Euro-Amerikanische « »gleichberechtigt repräsentiert und ausbalanciert werden«. Thaddeus Herrmann, Redakteur des deutschen Musik-Magazins für elektronische Musik De:Bug, hört überwiegend arabische Musik. Robert Koch hört es zunächst genau so, bemerkt jedoch, dass die Musik zum

17 Für eine Auflistung von Maqam-Skalen und Hörbeispielen verweisen wir auf die exzellente Internet-Plattform MaqamWorld: http: //www. maqamworld.com. 
Ende hin elektronischer wird und somit auch westlicher. Florian Sievers hört ebenfalls mehr arabische Einflüsse und empfindet »die Elektronik und Jazz Elemente eher nur als Tragegerüst und Antriebsmittel«. Für Benjamin Schäfer klingt die melodische Gestaltung und Artikulation des Gesangs, insbesondere die Vibratotechnik, typisch orientalisch. Der Musikpädagoge und Musiker Michael Ahlers argumentiert, »dass >Wanabni< einen starken arabischen Duktus und Impetus hat«. Michael Rappes erster Impuls war »mehr arabisch «. »Beim zweiten Nachdenken«, hört er »Wanabni« als eine gelungene »hybride«Musik. Rappe wertet diese Musik als »Türöffner«: Durch diese »Transformation« hätten »ungeübtere« Ohren auf einmal eine Möglichkeit, sich »reinzuhören«. Der Schweizer Kulturförderer Mauro Abbühl lokalisiert sowohl Jubran als auch Hasler eher in der Nähe der AvantgardeMusik des Westens als irgendeiner arabischen Schule oder Tradition. Die Musikwissenschaftlerin Guilnard Moufarrej meint, dass Jubran und Hasler hier eine originelle, eigene Musik schaffen. In ihrer Essenz findet sie diese Musik aber eher westlich als arabisch. Der Musikwissenschaftler und Journalist Tarek Khoury hört »Wanabni« weder als arabisch noch als europäisch:

»On the one hand it appears very Arabic - more than other so called >oriental groover music, that I know. Mainly the lyrics (with vocals in the foreground, not just decorative phrases or a looped two-words-sample), the oriental kind of singing and some central musical themes (trumpet intro/oud at the end of >Wanabni< etc.) may take this effect. But this effect is probably stronger due to my western enculturation - someone, who grew up with oriental music, would describe the same tracks maybe as very or more European sounding. In the end I would say the music is chiefly as well European and Arabic as something else, something new. Both musicians, Jubran and Hasler, are interpreting their traditional roots in a modern way, then they mix these interpretations to one piece of music - the result is something new, Arabic and European and in another perspective non-Arabic and non-European.«

Seth Ayyaz, elektro-akustischer Musiker aus Indien britischer Herkunft, will die Musik nicht >arabisch < nennen. Er vermisst die

»nuances and complexity of (traditional) Arabic pitch and rhythm constructions (e.g., heterophonic textures, improvisations with maqam developments, layered, inflected and fluctuating tempo, accelerando/ralantando etc.)«.

Kamran Rastegar lehnt die Kategorien sarabisch sie dann doch zu verwenden: "I would say idealistically that the music draws significantly from Arabic cultural materials, but is treated through a prism that is informed by European/Western avant-garde philosophies and 
approaches. « Auch Martin Stokes verwehrt sich gegen die konstruierten Kategorien sarabisch < und seuropäisch<. Er sagt dennoch: »The fact that vocals are somehow at the centre roots the thing in a certain kind of Arab world aesthetic, anchors it if you will.« Die ägyptische Musikwissenschaftlerin Azza Madian definiert die Musik als »Arab music, talking to Western oriented listeners; it could be an early step of a hybrid style of Arab music. [...] The textual factor is important. The text being in Classical Arabic strengthens that it is Arab music."

All diese Aussagen zeigen illustrativ, das essentialistische, an Nationen gebundene Kulturbegriffe für aktuelle popmusikalische Phänomene und Entwicklungen nicht mehr greifen, aber dennoch immer wieder verwendet werden. Prozess-orientierte, relative und reflexive und quer gelagerte Begriffe können hier weiterhelfen. Die identifizierten Stile Jazz, Electronica/Elektronische Laptopmusik und Tarab/Maqam können demnach als kulturelle Teil- und auch - je nach Kontext - Subprogramme im Sinne von Schmidt und Jacke beschrieben werden, die sich in ihrer konkreten Anwendung durch die Künstler und in ihrer Rekonstruktion durch die Experten/Hörenden aus deren jeweiligen Perspektiven und Kontexten von ursprünglichen Regeln und Konventionen entfernen (wollen) und dennoch aus jeweiligen Kulturationen auch erst entstehen.

Der Test zeigt exemplarisch, wie unterschiedlich verschiedene Hörer ein Musikstück hören. Gleichzeitig stützen die Testhörer viele unserer in Kapitel 3.1 aufgestellten Vermutungen. Sie verfeinern unsere Analyse aber mit neuen Hörbeobachtungen - das genau ist der Sinn eines multi-lokalen Hörtests.

\title{
4. Reaktionen von Kamilya Jubran und Werner Hasler
}

\begin{abstract}
Abschließend und im Sinne des dialogischen Editierens wurden Kamilya Jubran und Werner Hasler mit den Ergebnissen des Hörtests konfrontiert (Interviews Jubran 20.9.2010 und Hasler 21.9.2010). Interessant waren dabei unter anderem ihre Reaktionen auf die ausgemachten Maqam-Gattungen. Jubran betont, sie singe über einen Maqam Huzam und nicht Maqam Hijaz: »It is not at all hijaz, the intervals are different. Some listeners might get the illusion of hearing hijaz, because there is an interval of 1 and $1 / 2$ between the 4th and the 5th note, and this does exist in hijaz. «Nach eigenen Angaben singt Jubran über die folgende Haupttonleiter: es (erhöht) ${ }^{18}-f-$
\end{abstract}

18 Ein Viertelton über dem es. 
$g-a s-h-c-d-e s$ (erhöht) - diese Skala entspricht Maqam Huzam mit den typischen Intervallen $\left(3 / 4,1,1 / 2,1 \frac{1}{2}, 1 / 2,13 / 4\right)$. Jubran interpretiert Maqam Huzam nun aber anders, in dem sie das as als erste Tonebene denkt. Jubran und Hasler musizieren demnach über folgende Hauptskala: as (1. Ebene) $-h$ (2. Ebene) $-c$ (3. Ebene) $-d$ (4. Ebene) - es erhöht (5. Ebene) $-f$ (6. Ebene) $-g$ (7. Ebene) - as (1. Ebene). Jubran sagt es letztlich klar: Sie verwendet nicht alle Töne der dominanten Huzam-Skala. Zum Beispiel singt sie auf der ersten Tonebene eher $a$ statt as, und auch das $e$ scheint ebenso präsent wie das erhöhte es. Sie folgt den Prinzipien der MaqamMusik nur teilweise - das ist auch der Grund, warum Testhörer Martin Stokes dem Maqam nicht hat folgen können (vgl. 3.3.2). Wir hören hier auch die durch das ganze Stück andauernden tonalen Annäherungen und Reibungen zwischen der arabischen Sängerin und dem Schweizer Trompeter. Hasler betont dieses »unterschiedliche Intonieren« (Hasler 2010) im Interview.

In der weiteren Diskussion weisen sowohl Hasler als auch Jubran auf die Stücke »Shams « und »Asra« auf der CD »Wanabni« hin. »Shams« basiert auf einer Skala, die Hasler aus drei Obertonreihen konstruiert hat. »Asra« basiert über weite Strecken auf einer Ganztonskala. »The scale in >Asra< has no relation to any Arabic scale«, erklärt Jubran: »Werner gave me examples from Schönberg and Messiaen to listen to, and I tried to adapt these whole scale intervals to my singing. It was a great experience. « Werner Hasler war vom Resultat überrascht: »Bei uns heißt es immer, Ganztonskalen klingen kalt. Bei Kamilya wirken sie warm, und sie klingen plötzlich extrem >arabisch « « Ob unsere Hörtest-Personen diese beiden Stücke auch als »arabisch « eingeschätzt hätten, ist zwar zu vermuten, aber letztlich offen. Fakt ist, »Asra« und »Shams« haben von den Skalen her sehr wenig mit arabischer Musik zu tun. Für Hasler steht in der Zusammenarbeit mit Jubran ein Aspekt im Zentrum:

»Ich will neue Wege finden und außerhalb der üblichen funktionsharmonischen Abläufe improvisieren. Diese werden selbst im modalen Jazz nämlich immer noch mitgedacht. Wie improvisiert du in einem Modus, ohne harmonisch zu denken, so lautete letztlich meine Forschungsfrage.« 


\section{Schlussfolgerungen aus den Hörtests}

\subsection{Experten/Hörende}

Einige Hörerinnen und Hörer wiesen darauf hin, dass für die kulturelle Zuordnung einer Musik entscheidend ist, in welcher Sprache sie gesungen wird. Die Musik kann auf diesen tiefen Ebenen noch so hyper-kulturell sein, ist sie arabisch gesungen, wird sie mit grosser Bestimmtheit als multikulturell, interkulturell - oder im Extremfall transkulturell - wahrgenommen, und zwar wechselseitig. Im Hörtest wurde das an verschiedenen Stellen klar. Die meisten deutschen Hörer ordneten »Wanabni« wegen der Stimme und der Oud der arabischen Welt zu - oder zumindest der Weltmusik. Hieran wäre im Weiteren zu analysieren, wieso die Verortung und Einordnung der jeweiligen Herkünfte bzw. schwerpunktartigen Wurzeln der Musik im Gegenüber vermutet werden, also das jeweils sandere Kulturprogramm gewichtet wird, von Experten wie auch den Künstlern selbst. Evtl. zeichnet sich hier eine kulturprogrammlich tolerante Moderation im Sinne von Schmidt (1994, 2004) und Jacke (2004, 2009) insofern ab, als dass gewissermaßen das eigene Kulturprogramm ausgeblendet und das Augenmerk eher auf Unterschiede, Abweichungen und das jeweils $>$ Fremde - gelegt wird. Nichtsdestotrotz müssten diese Aspekte erst noch in einem (auch um unvorbelastete >normale Hörende) erweiterten Sample abgefragt und diskutiert werden.

\subsection{Die kulturellen Modi von »Wanabni«}

Fassen wir die konkreten Analysen noch einmal kurz zusammen: Aus musikalischer Sicht werten wir »Wanabni« zunächst einmal als eher transkulturell. Bestimmte Prinzipien aus Maqam/Tarab und Jazz/Electronika sind klar vorhanden, werden aber auf das im Moment klingende Material angepasst Hasler sucht laufend die passenden Tonlagen und Klänge zum Gesang von Kamilya Jubran. Und Jubran weiß, dass sie einen Maqam nicht komplett ausformulieren kann, wenn »Wanabni« als Ganzes funktionieren soll. »Wanab$\mathrm{ni} \ll$ fungiert allerdings nicht in den Modi multikulturell und interkulturell. Dazu formulieren beide Musiker zu viel um. Hyperkulturell ist »Wanabni « zunächst auch nicht, weil eben die Wurzeln des klingenden Materials hörbar bleiben.

Das Beispiel zeigt aber auch: Die Modi von multikulturell bis popkulturell können in der Musik selber auf verschiedenen Ebenen unterschiedlich gedeutet werden. Eine mögliche Leseart könnte demzufolge wie folgt lauten: 
»Wanabni« wirkt multikulturell, wenn man nur kurz hinhört und Form und Aufbau von »Wanabni« ignoriert: Dann nämlich rezitiert Jubran im Stil von Ataba-Sängern in der Bilad al Sham Region den Text und Hasler begleitet sie dezent als Jazz-Electronica Musiker - die beiden »Kulturen« stehen nebeneinander; »Wanabni« wirkt interkulturell, wenn Jubran zu Beginn des Stückes über Maqam Huzam singt und Hasler sie auf der Trompete improvisierend begleitet - die beiden »Kulturen« kommunizieren miteinander; »Wanabni« wirkt transkulturell, wenn Jubran den Ablauf der Maqam-Musik zwar imitiert, aber doch verändert, um mit Hasler in einen intensiven musikalischen Dialog zu treten. Im superkulturellen Modus steht »Wanabni« gar nicht, im popkulturellen Modus kaum einmal: Verweise aus Maqam/Tarab und Jazz/Electronika bleiben immer spürbar. Grundsätzlich kommunizieren die beiden Musiker deutlich mit dem jeweiligen Kanon ihrer musikalischen >Herkunfts<-Genres - Jubran hat den arabischen Gesang und das Oud-Spiel von ihrer Musikerfamilie gelernt und Werner Hasler wurde an der Schweizer Jazzschule in Bern ausgebildet. Die beiden Musiker spielen mit den Prinzipien dieser Traditionsströme und sie modulieren, irritieren sie. Sie brechen aber auch nicht alle Verbindungen ab. Als popkulturelle Spurenelemente können allerdings gegebenenfalls die Delay-Effekte oder Dub-Bässe gelesen werden, die »Wanabni « an verschiedenen Stellen beigemischt werden. Sie klingen komplett losgelöst von ihren ursprünglichen Kontexten und verbinden im wahrsten Sinn spielerisch musikalische Referenzen und Herkünfte und erschaffen damit neue Identitäten in einem Dazwischen.

Sowohl in ihren Selbstbeschreibungen als auch in ihrer Antizipation möglicher Kontexte, Geschichten und auch hinsichtlich ihrer Publika legen Jubran und Hasler großen Wert auf eine Verankerung in ihren je eigenen Kulturprogrammen. Gleichzeitig sind sie offen für Neues. ${ }^{19}$ Werner Hasler selbst fasst alle Diskussionen letztlich schön zusammen:

»Kamilya singt in arabischer Sprache mit arabischem Ausdruck und spielt Oud - das Hauptinstrument der arabischen Musikkultur. Ich spiele Trompete - komme vom Ausdruck her eher aus der Jazztradition - und Electronica, für mich ein unreglementiertes, freies Experimentierfeld, das in der europäischen Tradition der Maschineninstrumente steht. Man könnte sagen, Gesang und Sprache seien immer der zentrale Identifikationspunkt. Die $\mathrm{Mu}-$ sik an und für sich nährt sich aber nicht innerhalb von festen Schranken klar abgegrenzter Musiktraditionen. Wir schöpfen aus vielen Gefäßen und versuchen dabei klischierte Musik zu vermeiden, so kommt es, dass weder Araber noch Europäer die Musik gänzlich als ihrer Kultur zugehörig betrachten, das

19 Das lässt sich an allen hier geführten Interviews mit Jubran und Hasler klar belegen. 
ist ja genau, was wir als Duo sind. Gleichzeitig empfinden einige Europäer meinen Beitrag als Katalysator, weil sie die Feinheiten und Anspielungen auf die arabischen Wurzeln nicht erkennen können, sich aber in der Sonorität meines Instrumentariums und der Spielweise wiederfinden, was die Ferne überbrückt. Gleichzeitig kann man nicht sagen, dass sich Europäer per se in elektronischer Musik wiedererkennen, auch vor europäischem Publikum gerade in Frankreich - kann es passieren, dass einigen Leuten der arabische Gesang näher ist als die Electronica. Ich komme darauf zurück, was ist arabische oder europäische Musik heute? Der Baum hat viele Äste.«

\section{Selbstreflexion}

Die Studie zeigt in jeder Hinsicht die Vorzüge und Problematiken eines multiperspektivischen Ansatzes zur Erforschung populärer Musik, nämlich den Erkenntnisgewinn durch die Integration sowohl musik- als auch medienkulturanalytischer Blickpunkte und Methoden sowie die damit verbundene teilweise Unübersichtlichkeit der Ansätze, Methoden und Ergebnisse. Fest steht in jedem Fall das autologische Problem, dass auch die Forschenden im Team und mit ihren unterschiedlichen Perspektiven nicht aus dem Spiel der jeweiligen Kulturprogramme entkommen, womit wir zu einem sehr ähnlichen Ergebnis wie Thompson/Balkwill 2010 in ihrer eher musikpsychologischen Studie gelangen - um noch einmal Schmidt selbstkritisch und pointiert zu zitieren:

»Kulturbeschreibung setzt eine Beschreibungskultur voraus, und Kulturtheorie ist stets auch eine Form kultureller Praxis. [...] Unser Blick auf Kultur ist notwendigerweise ethnozentrisch. Und das gilt auch für alle Versuche, ethnozentrische Vorurteile bewusst zu vermeiden (was kultürlich ein ethnozentrisches Vorurteil besonderer Art ist, das nicht zuletzt wegen seiner normativen Implikationen berüchtigt ist)« (Schmidt 2004: 70f.).

\section{Fazit}

»Wanabni« konnte in der Analyse durch ausgewählte Experten aller Stufen des popmusikalischen Kommunikationsprozesses, durch die Künstler selbst und durch die Verfasser der Studie in seinen kulturellen Modi beobachtet und eingeordnet werden. Das Stück erscheint dabei offensichtlich weder rein traditionell verwurzelt (einerlei, auf welchem Kontinent) noch komplett losgelöst und beliebig vermengt. Die Musiker fühlen sich von Jazz, 
elektronischer Musik, Tarab und Maqam inspiriert und gleichzeitig mit ihrem jeweiligen Publikum in ihren verschiedenen Heimatländern und -Szenen verbunden.

Die Analysen sowie die Antworten und Wertungen aus dem Hörtest haben gezeigt, dass Kamilya Jubran und Werner Hasler gleichberechtigt zusammenarbeiten und sich beide stark mit dem musikalischen Traditionsstrom Tarab/Maqam befassen. „Wanabni« ist kein oberflächliches musikalisches Produkt, sondern das Resultat einer langfristigen musikalischen Annäherung und Begegnung. Genau hier liegen Sinn und Stärke eines multilokalen Hörtests und der Einordnung von Musikstücken in multi- bis popkulturelle Modi auf Grundlage eines weit gefassten, abstrakten Kulturkonzepts. Dieser methodisch-theoretische Ansatz lässt uns das musikalische Verarbeiten von musikalischem Material (hier Traditionsströme) genau und aus verschiedenen Perspektiven beobachten. Gleichzeitig kann er die Hierarchien zwischen den Musikern (innerhalb eines Projektes oder einer Band) aufdecken - gerade bei Projekten zwischen Musikern aus Europa/USA und Musikern aus Afrika/Asien/Lateinamerika scheint eine solche Untersuchung der projektinternen Machtverhältnisse besonders wichtig. Diese Wichtigkeit wird denn auch in verschiedenen Hörtestantworten, in den Reaktionen des Kairoer Publikums (diese Musik sei modern und gleichzeitig sehr arabisch) und im Konzept der »Alternativen Modernität« (Gaonkar 2001) immer wieder angedeutet.

Eine solche Musikanalyse ist bloß ein Teil des Ganzen. Weiterführende Studien könnten Kamilya Jubran und Werner Hasler stärker in sozialen, ökonomischen bis hin zu politischen Kontexten verorten. Eine anschließende, ausgeweitete Analyse könnte in die Richtung einer Forderung des Musikethnologen Veit Erlmann (1995) zielen. Dieser schlägt vor, Musik nach der Art und Weise zu untersuchen, in der die Geschichte eines kulturellen, sozial-politischen Kontexts in die Musik eingeschrieben ist. Wir würden sehen: Jubran und Hasler agieren und reagieren auch aus nicht-musikalischen Beweggründen heraus. Sie agieren in einem komplexen Netzwerk von Veranstaltern, Agenturen, Kulturförderern und Medien und versuchen, ihre Karrieren voranzutreiben. Nach Binas-Preisendörfer (2008: 11) wäre es eine Aufgabe für Ethnologen, die Interaktion dieser Einflüsse zu rekonstruieren und die daraus resultierenden ästhetischen Ideen zu fokussieren und $\mathrm{zu}$ reflektieren. 


\section{Literatur}

Ahlers, Michael / Jacke, Christoph (2010). »Kopulations-Kulissen: Ergebnisse und Forschungsperspektiven einer explorativen Studie zu Selektions- und Nutzungsbedingungen von Popmusik in erotischen und sexuellen Kontexten. «In: Thema Nr. 1. Sex und populäre Musik. Hg. Dietrich Helms u. Thomas Phleps (= Beiträge zur Popularmusikforschung 37). Bielefeld: Transcript, S. 201-228.

Appadurai, Arjun (2003). Modernity at Large: Cultural Dimensions of Globalization. Minneapolis, London: University of Minnesota Press.

Appadurai, Arjun (2009). Die Geographie des Zorns. Frankfurt/M.: Suhrkamp.

Asmar, Sami (1999). »Fairouz: a Voice, a Star, a Mystery.«In: Al Jadid Magazine 27, http://www.aljadid.com/music/fairouza_voice_star_mystery.html (Zugriff: 15.3.2011).

Assmann, Jan (1992). Das kulturelle Gedächtnis. Schrift, Erinnerung und politische Identität in frühen Hochkulturen. München: Beck.

Bergmann, Matthias / Jahn, Thomas / Knobloch, Tobias / Krohn, Wolfgang / Pohl, Christian / Schramm, Engelbert (2010). Methoden transdisziplinärer Forschung. Ein Überblick mit Anwendungsbeispielen. Frankfurt/M., New York: Campus.

Binas-Preisendörfer, Susanne (2010). Klänge im Zeitalter ihrer medialen Verfügbarkeit. Popmusik auf globalen Märkten und in lokalen Kontexten. Bielefeld: Transcript.

Binas-Preisendörfer, Susanne (2008). »Rau, süßlich, transparent oder dumpf Sound als eine ästhetische Kategorie populärer Musikformen. Annäherung an einen populären Begriff.«In: PopScriptum 10, S. 1-15, http://www2.hu-berlin. $\mathrm{de} / \mathrm{fpm} /$ popscrip/themen/pst10/pst10_binas.htm (Zugriff: 21.9.2011).

Burkhalter, Thomas (2003). »Leise Musiker in einer lauten Stadt: Unabhängige Musiker im 16-Millionen Moloch Kairo. « In: Norient. Independent Network for Local and Global Soundscapes, http:// http://norient.com/stories/musikszenekairo (Zugriff: 15.3.2011).

Burkhalter, Thomas (2006). »Un regard personnel sur sa propre culture.« In: Norient. Independent Network for Local and Global Soundscapes, http: //norient.com/stories/wammeed (Zugriff: 15.3.2011).

Burkhalter, Thomas (2009). Challenging the Concept of Cultural Difference: $\gg L O-$ cality« and »Place in the Music of Contemporary Beirut. Bern: Universität Bern (unveröffentlichte Dissertationsschrift).

Cascone, Kim (2003). »Deterritorialisierung, historisches Bewusstsein, System. Die Rezeption der Performance von Laptop-Musik.«In: Soundcultures. Über elektronische und digitale Musik. Hg. v. Marcus S. Kleiner u. Achim Szepanski. Frankfurt/M.: Suhrkamp, S. 101-106.

Diederichsen, Detlef (2010). „Cibelle. Oder Du nimmst ein Raumschiff nach Sonstwo. «n: Spex. Magazin für Popkultur, H. 326 (Mai/Juni), S. 20-22.

Elflein, Dietmar (2010). Schwermetallanalysen: Die musikalische Sprache des Heavy Metal ( = texte zur populären musik 6). Bielefeld: Transcript.

Erlmann, Veit (1995). »Ideologie der Differenz: Zur Ästhetik der World Music.« In: PopScriptum 3, S. 6-29, http://www2.hu-berlin.de/fpm/popscrip/themen/ pst03/pst03010.htm (Zugriff: 15.3.2011).

Feld, Steven (1982). Sound and Sentiment: Birds, Weepings, Poetics, and Song in Kaluli Expression. Philadelphia: University of Pennsylvania Press. 
Freitag, Jan (2011). »Jenseits von Bollywood.«In: Die Zeit, H. 12 vom 17. März, S. 70 .

Gaonkar, Dilip Parameshwar (2001). Alternative Modernities. Durham: Duke University Press.

Gebesmair, Andreas (2009). »The Transnational Music Industry.« In: The Ashgate Research Companion to Popular Musicology. Hg. v. Derek B. Scott. Farnham, Burlington: Ashgate, S. 467-483.

Gobo, Giampietro (2008). Doing Ethnography. London u.a.: Sage.

Goer, Charis / Greif, Stefan / Jacke, Christoph (2010). »Poptheorie, Popkulturforschung und Literaturwissenschaft.« In: Literaturwissenschaft interdisziplinär. Hg. v. Lothar van Laak und Katja Malsch. Heidelberg: Synchron, S. 211-229.

Ha, Kien Nghi (2005). Hype um Hybridität. Kultureller Differenzkonsum und postmoderne Verwertungstechniken im Spätkapitalismus. Bielefeld: Transcript.

Hage, Ghassan (2003). Against Paranoid Nationalism: Searching for Hope in a Shrinking Society. Annandale: Pluto Press.

Han, Byung-Chul (2005). Hyperkulturalität. Kultur und Globalisierung. Berlin: Merve.

Hepp, Andreas (2006). Transkulturelle Kommunikation. Konstanz: UVK [UTB].

Ismaiel-Wendt, Johannes (2010). tracks'n'treks. Populäre Musik und postkoloniale Analyse. Bremen: Universität Bremen (unveröffentlichte Dissertationsschrift).

Jacke, Christoph (2004). Medien(sub)kultur. Geschichten - Diskurse - Entwürfe. Bielefeld: Transcript.

Jacke, Christoph (2009). Einführung in Populäre Musik und Medien. Münster: LIT.

Jacke, Christoph / Passaro, Sandra (2011). »>Pop Will Eat Itself. Will Pop Eat Itself? : Aktuelle Entwicklungen der transnationalen Popmusikindustrien. Eine Expertenbefragung. «In: »They Say I'm Different ...«. Popularmusik, Szenen und ihre AkteurInnen. Hg. v. Wolfgang Fichna und Rosa Reitsamer. Wien: Löcker, S. 95-115.

Klein, Gabriele / Friedrich, Malte (2003). „Globalisierung und die Performanz des Pop. «In: Popvisionen. Links in die Zukunft. Hg. v. Klaus Neumann-Braun, Axel Schmidt und Manfred Mai. Frankfurt/M.: Suhrkamp, S. 77-102.

Lull, James (1992). »Popular Music and Communication: An Introduction.« In: Popular Music and Communication. Hg. v. James Lull. Newbury Park: Sage (2. Aufl.), S. 1-32.

Lull, James (2000). Media, Communication, Culture: A Global Approach. New York: Columbia University Press (2. Aufl.).

Lull, James (2002). "Superkultur.«In: Grundlagentexte zur transkulturellen Kommunikation. Hg. v. Andreas Hepp und Martin Löffelholz. Konstanz: UVK [UTB], S. 750-773.

Marcus, George E. (1995). »Ethnography in/of the World System: The Emergence of Multi-sited Ethnography. «In: Annual Review of Anthropology 24, S. 95-117.

Meyer, Andreas (2007). »Heimatklänge - Lokale Popmusik und transkulturative Prozesse. «In: Pop Insights. Bestandsaufnahmen aktueller Pop- und Medienkultur. Hg. v. Thomas Krettenauer und Michael Ahlers. Bielefeld: Transcript, S. 35-46.

Mittelstraß, Jürgen (1998). Die Häuser des Wissens. Wissenschaftstheoretische Studien. Frankfurt/M.: Suhrkamp.

Muncey, Tessa (2010). Creating Autoethnographies. London u.a.: Sage.

O'Reilly, Karen (2009). Key Concepts in Ethnography. London u.a.: Sage.

Peterson, Richard A. / Bennett, Andy (2004). »Introducing Music Scenes. «In: Music Scenes. Local, Translocal, and Virtual. Hg. v. dens. Nashville: Vanderbilt University Press, S. 1-15. 
Pfleiderer, Martin (1998). Zwischen Exotismus und Weltmusik. Zur Rezeption asiatischer und afrikanischer Musik im Jazz der 60er und 70er Jahre. Karben: CODA.

Racy, Ali Jihad (1996). »Heroes, Lovers, and Poet-Singers: The Bedouin Ethos in the Music of the Arab Near-East. «In: The Journal of American Folklore 434, S. 404424.

Racy, Ali Jihad (2003). Making Music in the Arab World: The Culture and Artistry of Tarab. Cambridge: Cambridge University Press.

Randeria, Shalini / Eckert, Andreas (2009). »Geteilte Globalisierung.«In: Vom Imperialismus zum Empire. Hg. v. dens. Frankfurt/M.: Suhrkamp, S. 9-31.

Rosengren, Karl Erik (2002). »Internationale und interkulturelle Kommunikation.« In: Grundlagentexte zur transkulturellen Kommunikation. Hg. v. Andreas Hepp u. Martin Löffelholz. München: UVK/UTB, S. 37-66.

Rutten, Paul (1994). "Lokale Musik und der internationale Marktplatz.« In: PopScriptum 2, S. 31-45.

Schmidt, Siegfried J. (1994). Kognitive Autonomie und soziale Orientierung. Konstruktivistische Bemerkungen zum Zusammenhang von Kognition, Kommunikation, Medien und Kultur. Frankfurt/M.: Suhrkamp.

Schmidt, Siegfried J. (2004). Unternehmenskultur. Die Grundlage für den wirtschaftlichen Erfolg von Unternehmen. Weilerswist: Velbrück.

Shannon, Jonathan Holt (2006). Among the Jasmine Trees: Music and Modernity in Contemporary Syria. Middleton: Wesleyan University Press.

Solomon, Thomas (2009). »Berlin - Frankfurt - Istanbul: Turkish Hip-Hop in Motion. «In: European Journal of Cultural Studies 12, H. 3, S. 305-327.

Steinholt, Yngvar Bordewich (2005). Rock in the Reservation: Songs from the Leningrad Rock Club. New York: Mass Media Music Scholars' Press.

Stokes, Martin (1992). The Arabesk Debate: Music and Musicians in Modern Turkey. Oxford: Claredon Press.

Stokes, Martin (2000). »East, West, and Arabesk. «In: Western Music and Its Others: Difference, Representation, and Appropriation in Music. Hg. v. Georgina Born. Berkeley: University of California Press, S. 213-233.

Stone, Christopher (2008). Popular Culture and Nationalism in Lebanon: The Fairouz and Rahbani Nation. New York: Routledge.

Tagg, Philip (2009). »Music Analysis for >Non-Musos $<$ : Popular Perception as a Basis for Understanding Musical Structure and Signification. “ Conference »Popular Music Analysis«. University of Cardiff (Conference Paper); updated version: http: / /www.tagg.org/xpdfs/CardiffLBH2.pdf (Zugriff: 15.3.2011).

Tagg, Philip / Clarida, Bob (2003). Ten Little Tunes: Towards a Musicology of the Mass Media. New York, Montreal: The Mass Media Music Scholars' Press.

Terkessidis, Mark (2006). »Distanzierte Forscher und selbstreflexive Gegenstände. Zur Kritik der Cultural Studies in Deutschland.«In: Kulturschutt. Über das Recycling von Theorien und Kulturen. Hg. v. Christoph Jacke, Eva Kimminich und Siegfried J. Schmidt. Bielefeld: Transcript, S. 148-162.

Terkessidis, Mark (2008). "Globale Kultur in Deutschland: Der lange Abschied von der Fremdheit. « In: Kultur - Medien - Macht. Cultural Studies und Medienanalyse. Hg. v. Andreas Hepp und Rainer Winter. Wiesbaden: VS (4. Aufl.), S. 311-325.

Terkessidis, Mark (2010). Interkultur. Frankfurt/M.: Suhrkamp.

Thompson, William Forde / Balkwill, Laura-Lee (2010). »Cross-Cultural Similarities and Differences. "In: Handbook of Music and Emotion. Theory, Research, Applications. Hg. v. Patrik N. Juslin und John A. Sloboda. Oxford: OUP, S. 755-788.

Touma, Habib Hassan (1998). Die Musik der Araber. Wilhelmshaven: Noetzel. 
Wallis, Roger / Malm, Krister (1987). »The International Music Industry and Transcultural Communication. «In: Popular Music and Communication. Hg. v. James Lull. Newbury Park: Sage, S. 112-137.

Weinrich, Ines (2006). Fairuz und die Brüder Rahbani. Musik, Moderne und Nation im Libanon. Würzburg: Ergon Verlag.

Welsch, Wolfgang (1994). »Transkulturalität. Lebensformen nach der Auflösung der Kulturen.«In: Dialog der Kulturen. Die multikulturelle Gesellschaft und die Medien. Hg. v. Kurt Luger und Rudi Renger. Wien, St. Johann im Pongau: Österreichischer Kunst- und Kulturverlag, S. 147-169.

Welsch, Wolfgang (2010). "Standbeine dürfen nicht zum Klumpfuß werden. Wolfgang Welsch im Gespräch über eine transkulturell orientierte Gesellschaft - und wie Musik Menschen zusammenführen kann.« In: Musikforum 8:1, S. 8-12.

Wicke, Peter (2001). Von Mozart zu Madonna. Eine Kulturgeschichte der Popmusik. Frankfurt/M.: Suhrkamp.

Žižek, Slavoj (2003). Ein Plädoyer für die Intoleranz. Wien: Passagen (3. Aufl.).

\title{
Interviews der Autoren mit den Künstlern
}

Werner Hasler 01.04.2002

Kamilya Jubran 14.04.2002

Werner Hasler 04.08.2010

Kamilya Jubran 11.08.2010

Kamilya Jubran 20.09.2010

Werner Hasler 21.09.2010

\begin{abstract}
This article discusses the results of a multi-local listening test conducted with listeners in Europe and the Arab world. We asked musicians, musicologists, music lovers, and students to listen to the piece »Wanabni«, written and played by the Palestinian oud player Kamilya Jubran and the Swiss trumpet player and electroacoustic musician Werner Hasler. The listeners were requested to discuss this piece of music on different levels: the performance itself (constructional competence), the musical references (receptional competence), and the musical and non-musical contextualisations and positionings (metatextual and metacontextual discourse). The article highlights the multiple meanings that a single piece of music can generate, focussing on different listeners with different interlocal/intercultural/ interprofessional experiences and engagements. The article also aims to present one possible way of analysing multi-, trans- or hyper-cultural music and, moreover, its reception, in today's increasingly digitised and globalised music world.
\end{abstract}




\section{Anhang 1: Der Fragebogen / Questionnaire Listening Test}

\section{First Impressions}

What are your first impressions/associations when you hear this music?

\section{References}

Is this music inspired by specific bands, musicians, composers, and songs?

Are there non-musical references?

\section{Musical Qualities}

Do you like the music? Why? Or why not?

Do you think it is original, innovative, special, just an imitation, ...?

Do you think this music is in its essence more »European« or more »Arabic «?

Or the opposite? Or something else?

Melodies, Rhythms, Forms

How is the music structured and arranged?

Do you hear specific tonal structures (harmonies, chord progressions, chords, keys, modalities, scales, quartertones, ornamentations...) (please indicate with time codes)?

Do you hear specific time structures (rhythms, time patterns, ...)

Do you hear something rather specific, unique, surprising?

\section{Instruments, Samples, Sounds}

Do you recognize specific sounds: instruments, samples, electronic sounds, etc.?

Do you hear specific technical aspects (types of instruments, guitar strings, types of microphones, amplifiers, effect devises, mix and mastering)?

How are these sounds produced: live instruments, different sound software, from sample databases (in specific sound software, synthesizers, etc.)?

How would you describe the production itself?

How do you hear timbres, textures, sounds, volumes of this music?

\section{Musical Performance / Interpretation}

How would you describe the way the different players use their »instruments «? How exactly do these musicians arrange, interconnect and play their sonic material?

Do you hear a specific/typical or unusual treatment of the instruments/sounds? How do they treat sound (reverb, delay, phrasing, distortion, etc.)?

Do you hear references to other musicians in the way the musicians/artists treat sound(s), their voice, or their instruments? 


\section{Anhang 2: Liste aller Teilnehmer des Hörtests}

*BS: Benjamin Schäfer > Musiker, Popmusikforscher, Mitarbeiter Fach Musik/Populäre Musik und Medien Universität Paderborn (Produktion, Rezeption, Weiterverarbeitung)

*EW: Eckhard Wiemann > Pianist, Musiker, Produzent, Lehrender Fach Musik/Populäre Musik und Medien Universität Paderborn (Produktion, Rezeption, Weiterverarbeitung)

*JB: Jochen Bonz, > Journalist, Popkultur/Musikforschender, Ethnologe/Soziologe, Universität Gießen (Distribution, Rezeption, Weiterverarbeitung)

*AB: Mauro Abbühl > Kulturförderer, Bern, ArtLink (Rezeption, Weiterverarbeitung, Distribution)

*MA: Michael Ahlers > Musiker, Produzent, Popmusikforscher, Musikpädagoge, Mitarbeiter Fach Musik/Populäre Musik und Medien/Fach Musik Universität Paderborn, Professor für Musikpädagogik/Digitale Medien Universität Augsburg (Produktion, Rezeption, Weiterverarbeitung)

*SA. Seth Ayyaz > Musiker, Musikwissenschaftler, City University London und Zenith Foundation (Produktion, Rezeption, Weiterverarbeitung)

*KA: Kinan Azmeh > Musiker, Damaskus (Produktion)

*RB: Roger Behrens > Musiker, Journalist, Mitherausgeber Testcard, Popmusik/ -kultur-Philosoph, Lehraufträge u.a. an den Universitäten Weimar, Hamburg, Lüneburg und Paderborn (Produktion, Rezeption, Weiterverarbeitung)

*PB: Paul Bonomo (aka Snax, Tony Amherst, Captain Comatose) > Produzent, Musiker, DJ, Labelinhaber Random Rec., Berlin (Produktion, Rezeption, Weiterverarbeitung, Distribution)

*ME: Manuel Engelbertz > Kulturwissenschaftler und freier Musikjournalist, Magazin Friedrich, Berlin (Rezeption, Weiterverarbeitung)

*SF: Sven Fortmann > Journalist Lowdown-Magazin, Berlin (Rezeption, Weiterverarbeitung)

${ }^{*}$ CG: Christine Giese > DJ Suzi Wong, Journalistin (Radio), Berlin (Rezeption, Weiterverarbeitung)

*TH: Thaddeus Herrmann > Journalist/Redakteur De:Bug-Magazin, Label City Centre Offices, DJ, Berlin (Produktion, Rezeption, Weiterverarbeitung, Distribution)

*OK: Olaf Karnik > Musiker, DJ, Journalist, Kurator, Popmusikforschender, Fach Musik/Populäre Musik und Medien Universität Paderborn (Produktion, Distribution, Rezeption, Weiterverarbeitung)

*TK: Tarek Khoury > Musikwissenschaftler, Produzent und Journalist (Radio), Köln (Produktion, Rezeption, Weiterverarbeitung) 
*RK: Robert Koch > Produzent, Musiker, Bands Jahcoozi, The Tape, Robot Koch, Label, DJ, Berlin (Produktion, Rezeption, Weiterverarbeitung, Distribution)

*UL: Uli Lettermann > Saxophonist, Musiker, Lehrender Fach Musik/Populäre Musik und Medien Universität Paderborn (Produktion, Rezeption, Weiterverarbeitung)

*NL: Neil van der Linden > Musikethnologe und Kulturproduzent, Amsterdam (Rezeption, Weiterverarbeitung)

*AM: Azza Madian > Musikwissenschaftlerin, Bibliothek von Alexandria und Kairo Symphony Orchestra (Rezeption, Weiterverarbeitung)

*MK: Karima Mansour > Zeitgenössische Tänzerin, Kairo (Produktion, Weiterverarbeitung)

*GM: Guilnard Moufarrej > Musikethnologin, UCLA Los Angeles (Rezeption, Weiterverarbeitung)

*ZN: Ziad Nawfal > Radiojournalist, Radio Lebanon, Beirut (Rezeption, Distribution)

*KN: Kristina Nelson > Kulturvermittlerin, Kairo (Rezeption, Weiterverarbeitung)

*MP: Martin Pfleiderer > Saxophonist, Professor für Geschichte des Jazz und populärer Musik, Hochschule für Musik Franz Liszt, Weimar (Produktion, Rezeption, Weiterverarbeitung)

*MR: Michael Rappe > Musiker, Produzent, DJ, Veranstalter, Professor für Populäre Musik, Hochschule für Musik und Tanz Köln (Produktion, Distribution, Rezeption, Weiterverarbeitung)

*KR: Kamran Rastegar > Musiker, Literaturwissenschaftler, Department of German, Russian \& Asian Languages, Tufts University, Medfort (Produktion, Rezeption, Weiterverarbeitung, Distribution)

*AR: Akram Rayess > Musikwissenschaftler, Beirut (Rezeption, Weiterverarbeitung)

*SA: Mustafa Said > Oud-Spieler, Kairo (Produktion)

*AS: Ahmad El Sawy > Oud-Spieler, Kairo (Produktion)

*SC: Manuel Schottmüller > Booker, »SSC Booking«, Düsseldorf (Rezeption, Weiterverarbeitung)

*JS: Jonathan Shannon > Ethnologe, Anthropology Faculty, Hunter College of the City of New York (Rezeption, Weiterverarbeitung)

*FS: Florian Sievers > Journalist, Berlin (Rezeption, Weiterverarbeitung)

*MS: Martin Stokes > Musikethnologe, lecturer in Ethnomusicology, Oxford University (Rezeption, Weiterverarbeitung)

${ }^{*} \mathrm{MW}$ : Michael Wentzlaff > Schauspieler, Berlin (Rezeption, Weiterverarbeitung) 